\title{
Characteristics of Endophthalmitis after Cataract Surgery in the United States Medicare Population
}

\author{
Emily W. Gower, $\mathrm{PhD}^{1,2}$, Lisa J. Keay, $\mathrm{PhD}^{3}$, Dianne E. Stare, $\mathrm{MPH}^{2}$, Pallavi Arora, MD $^{2}$, \\ Sandra D. Cassard, ScD², Ashley Behrens, MD $^{2}$, James M. Tielsch, PhD $^{4}$, and Oliver D. \\ Schein, MD, MPH ${ }^{2}$
}

${ }^{1}$ Departments of Epidemiology and Ophthalmology, Wake Forest School of Medicine, WinstonSalem, North Carolina ${ }^{2}$ Wilmer Eye Institute, Johns Hopkins University School of Medicine, Baltimore, Maryland ${ }^{3}$ The George Institute for Global Health, University of Sydney, Sydney, Australia ${ }^{4}$ George Washington University School of Public Health and Health Services, Washington, District of Columbia

\begin{abstract}
Purpose-Endophthalmitis is a rare but sight-threatening infection after cataract surgery. Roughly one third of eyes remain blind after treatment. We report United States population-based data on microbiological investigations and treatment patterns plus risk factors for poor outcomes.

Design-Retrospective cohort study.

Participants-Medicare beneficiaries from 5 states in whom endophthalmitis developed within 6 weeks after cataract surgery in 2003 and 2004.

Methods-We identified endophthalmitis cases occurring after cataract surgery using Medicare billing claims. We contacted treating physicians and requested they complete a questionnaire on clinical and microbiological data and submit relevant medical records. Two independent observers reviewed materials to confirm that cases met a standardized definition.
\end{abstract}

Main Outcome Measures-Positive culture results, vitrectomy status, microbiology spectrum, and final visual acuity.

Results-In total, 615 cases met our case definition. Initial visual acuity was counting fingers or worse for $72 \%$. Among 502 cases with known culture results, 291 (58\%) had culture positive results. Twelve percent had positive results for streptococci. More than $99 \%$ of cases were treated with intravitreal vancomycin. Vitrectomy was performed in 279 cases (45\%), including 201 cases

Correspondence: Emily W. Gower, PhD, Wake Forest Health Sciences, Medical Center Boulevard, Winston-Salem, NC 27106. egower@wakehealth.edu.

Presented in part at: Association for Research in Vision and Ophthalmology Annual Meeting, May 2010, Fort Lauderdale, Florida.

Financial Disclosure(s): The author(s) have no proprietary or commercial interest in any materials discussed in this article.

Author Contributions: Conception and design: Gower, Tielsch, Schein

Analysis and interpretation: Gower, Arora, Cassard, Behrens, Tielsch, Schein

Data collection: Gower, Keay, Stare, Arora, Cassard, Behrens, Tielsch, Schein

Obtained funding: Gower, Schein, Tielsch

Overall responsibility: Gower, Keay, Stare, Arora, Cassard, Behrens, Tielsch, Schein 
with initial acuity better than light perception. Rates of vitrectomy varied across states, with California having the highest rate and Michigan having the lowest (56\% and 19\% of cases, respectively). Overall, $43 \%$ of individuals achieved visual acuity of $20 / 40$ or better. Poor initial acuity (adjusted odds ratio [OR], 1.08; 95\% confidence interval [CI], 1.04-1.12 per 0.10 logarithm of the minimum angle of resolution units), older age at diagnosis (OR, 1.22; 95\% CI, 1.03-1.45 per 5-year increase), and more virulent organisms were important predictors of poor final visual acuity. Cases with streptococci infection were 10 times more likely to have poor final acuity than coagulase-negative staphylococci cases (adjusted OR, 11.28; 95\% CI, 3.63-35.03). Vitrectomy was not predictive of final visual acuity (adjusted OR, 1.26; 95\% CI, 0.78-2.04).

Conclusions-Population-based data on the microbiology of acute postoperative endophthalmitis in the United States after cataract surgery are consistent with prior reports. Vitrectomy usage is higher than that recommended from the Endophthalmitis Vitrectomy Study, with no evidence of increased benefit.

Cataract surgery is the most commonly performed surgical procedure in the United States. Endophthalmitis remains a rare but serious complication of cataract surgery. Previous studies analyzing Medicare claims data report an incidence of 1.1 to 1.33 endophthalmitis cases per 1000 surgeries in 2003 and 2004, ${ }^{1} 1.6$ cases per 1000 surgeries from 1994 through $2006,{ }^{2}$ and 0.63 to 1.27 cases per 1000 surgeries from 2006 through $2011,{ }^{3}$ depending on how strictly endophthalmitis is defined. The Endophthalmitis Vitrectomy Study (EVS) is the only clinical trial to have evaluated the effects of vitrectomy and systemic antibiotics on visual outcomes among cases of endophthalmitis occurring after cataract surgery. The study reported no benefit from using systemic antibiotics and no benefit of vitrectomy in eyes with vision better than light perception (LP) at presentation. However, among patients with LP only, vitrectomy was associated with a 3-fold chance of improved visual acuity outcomes. ${ }^{4}$ A more recent study conducted in Australia reported similar findings related to the benefit of vitrectomy, but found that systemic antibiotics significantly improved final visual acuity. ${ }^{5}$ Within that study, however, $22 \%$ of 107 participants in the multivariate analysis were not treated with intravitreal antibiotics, which limits evaluation of the benefits of systemic antibiotics in addition to intravitreal antibiotics. The purpose of this study was to characterize the presenting features and causative organisms of a nationally representative sample of endophthalmitis cases over a 2-year period and to evaluate clinical management patterns and associated outcomes.

\section{Methods}

Data for this study derive from the Endophthalmitis Post-cataract Surgery study. The Endophthalmitis Post-cataract Surgery investigation was a case-control study that was conducted to evaluate risk factors for endophthalmitis occurring after cataract surgery within the United States. Five states were included: California, Texas, Florida, Michigan, and Illinois. We selected these states because they have high numbers of cataract surgeries ${ }^{6}$ and because their endophthalmitis rates for 2003 through 2004 were similar to the national average. ${ }^{1}$

We identified endophthalmitis cases using data from the Medicare billing database for 2003 through 2004. We received initial access to these files in 2007 because there is a lag between 
services provided and availability of the data for research purposes. All billing records for all Medicare patients (100\% sample) in which an International Disease Classification, Ninth Revision, code for endophthalmitis was used were provided by the Centers for Medicaid and Medicare Services. International Disease Classification, Ninth Revision, codes included 360.00 through 360.04. All cataract surgery claims for the same period also were provided as described previously. ${ }^{1}$ We presumed that the endophthalmitis was associated with cataract surgery if the first endophthalmitis claim was made within 42 days after cataract surgery.

The treating physician for each endophthalmitis case, typically a retinal specialist, was contacted by telephone or mail and asked to participate. Consenting physicians were sent a brief clinical data collection form and were asked to provide relevant chart notes for each case. When the retinal specialist could not be identified or declined to participate, consultation letters from the retinal specialist to the cataract surgeon were used to obtain as much information as possible to confirm case details.

Trained abstractors reviewed chart notes to complete any questions left blank on the clinical data collection form. Two ophthalmologists then independently reviewed all data for each patient to determine whether the patient met our endophthalmitis case definition. Individuals were considered to have endophthalmitis if hypopyon or vitreitis was present or if the patient received an intravitreal injection of antibiotics for presumed endophthalmitis within 42 days of the index cataract surgery. This process was followed over a 5-year period from 2007 through 2011.

Presenting acuity was based on the data recorded in the patient's medical records and defined as the acuity at the first visit for endophthalmitis. Final acuity was recorded based on the final visit for which the patient was being treated for endophthalmitis (i.e., when the symptoms resolved).

On occasion, the retinal specialist's office completed and returned the questionnaire without accompanying chart notes. In some of these cases, some signs of endophthalmitis were checked as present, whereas others were left blank, making it difficult to determine details of case presentation. We classify these cases as indeterminate and reported them as a separate group.

We analyzed these data using the SAS software version 9.2 of the SAS System for Linux (SAS Inc., Cary, NC). We performed bivariate comparisons using continuity-adjusted chisquare tests and used multivariate logistic regression to evaluate the association between a variety of covariates and a dichotomous outcome of visual acuity of 20/200 or worse versus 20/100 or better. We considered a $P$ value of less than 0.05 to be statistically significant.

The Johns Hopkins Medicine Institutional Review Board and the Privacy Board at the Centers for Medicare and Medicaid Services approved the research protocol. This study adhered to the tenets of the Declaration of Helsinki. 


\section{Results}

We identified 1268 cases of presumed endophthalmitis that occurred after cataract surgery among the Medicare population from 2003 through 2004 in the 5 states studied. These cases represent approximately $32 \%$ of all presumed endophthalmitis cases occurring after cataract surgery in the United States for that 2-year period. Attempts were made to contact the retinal specialist for 1213 cases. The retinal specialists for the remaining 55 cases were not contacted because we had already reached our target number of cases for the case-control study (results forthcoming) before contact was attempted.

\section{Case Confirmation and Demographics}

We received sufficient information to determine case status for 719 presumed endophthalmitis cases. The 2 case status reviewers concluded that $615(86 \%)$ of these individuals met our case definition for endophthalmitis occurring after cataract surgery. The remaining 104 did not meet the case definition and were excluded from further analyses. For an additional 11 presumed endophthalmitis cases, we did not receive sufficient information to classify them definitively as cases, and hence we excluded them from these analyses.

Demographic information for the 615 confirmed cases is provided in Table 1. The median age was 78 years (range, 65-101 years), and 12\% were not white. Individuals for whom we did not have case confirmation data were slightly younger (mean age, 77 years) and were more likely not to be white (17\%). The percent male was similar between those with data and those without case confirmation. Florida had the largest number of cases and Michigan had the fewest. Most patients (72\%) had visual acuity of counting fingers or worse at presentation, and 7 eyes had no LP. The median time to onset of symptoms was 4 days after surgery, and most cases (64\%) sought treatment from a retinal specialist within 1 week after cataract surgery (Table 1). Hypopyon was reported as present for $69 \%$ of cases, vitreitis was reported as present for $64 \%$, and $4+$ cell and flare was reported as present for $53 \%$. Followup time ranged from a single visit to more than 5 years. The median follow-up time was 83 days, and $78 \%$ were followed up for at least 30 days. A total of 293 known retinal specialists treated the 615 cases. The number of retinal specialists by state is as follows: California, 82; Florida 75; Illinois, 40; Michigan 34; and Texas, 62.

\section{Culture Results}

Culture results were known for 502 individuals, and the results for 291 of them were positive (58\% of cultured cases). Among the group with culture results available, culturepositive cases were more likely to have visual acuity of 20/200 or worse at presentation (92\% vs. $78 \% ; P<0.0001$; Table 2 ) and were more likely to undergo vitrectomy (59\% vs. $36 \% ; P<0.0001)$. Ninety-three percent of cultured organisms were gram positive. Coagulasenegative staphylococci (CNS) were the most commonly cultured organisms (45\% of positive cultures; Fig 1). We did not see a relationship between the time to presentation to a retinal specialist and organism cultured, and pathogen distribution was similar across race and between genders. Culture-positive cases were more likely than culture-negative cases to have a final acuity of $20 / 200$ or worse ( $38 \%$ vs. $27 \%$; $P=0.01)$. Among culturepositive cases with known organisms, those with streptococci were more likely than other culture- 
positive cases to have a final visual acuity of $20 / 200$ or worse ( $85 \%$ vs. $32 \%$; $P<0.001$ ). Gramnegative cases had visual outcomes more similar to those with streptococci infections than to other gram-positive cases (65\% with final visual acuity of 20/200 or worse compared with $37 \%$ of other culture-positive gram-positive cases; $P=0.04$ ).

\section{Vitrectomy}

Vitrectomy was performed for 279 cases (45\%). Eight-five percent of cases with initial acuity of LP and $43 \%$ of the 7 cases with no light perception (NLP) underwent vitrectomy. Vitrectomy was common in eyes with acuity better than LP (Table 3 ) and was performed in $28 \%$ of cases with acuity of 20/100 or better. Among cases managed with vitrectomy, a vitrectomy was performed within the first 2 days after seeking treatment from a retinal specialist for $75 \%$ and more than 1 week after initial treatment for $17 \%$. Use of vitrectomy varied across states. For cases with an initial acuity of LP, $77 \%$ to $91 \%$ of cases were treated with vitrectomy. The percent of cases with better than LP vision at presentation that was treated with vitrectomy ranged from 19\% of cases in Michigan to 56\% in California (Table 4). In multivariate logistic regression analyses predicting use of vitrectomy among cases with initial acuity better than LP, state where the procedure was carried out remained a significant predictor after adjusting for initial acuity, age, and type of organism cultured (data not shown). Retinal surgeons in California were 9 times more likely to use vitrectomy than were retinal surgeons in Michigan (adjusted odds ratio, 9.18; 95\% confidence interval, 3.05-27.62).

Seventy-six percent of cases with initial acuity of LP underwent immediate vitrectomy, and $9 \%$ underwent vitrectomy 2 days or more after initial presentation. Among cases with initial acuity better than LP, $28 \%$ underwent immediate vitrectomy and $13 \%$ underwent vitrectomy 2 days or more later, with most of the delayed vitrectomies occurring more than 1 week after initial presentation. More than one third of vitrectomies performed on patients with visual acuity of hand movements were performed more than 1 week after presentation.

Among cases with initial acuity of LP, there was no statistically significant difference in final acuity outcomes comparing the cases treated with vitrectomy versus those without vitrectomy (Table 5). Similarly, among cases with initial acuity better than LP, vitrectomy did not improve final visual acuity status. Approximately $23 \%$ of cases with no vitrectomy and those with a vitrectomy within the first 2 days had final acuity of 20/200 or worse. Among the cases with initial acuity better than LP, more than $50 \%$ of those who had a vitrectomy more than 2 days after diagnosis had a final acuity of 20/200 or worse. In multivariate analyses adjusting for initial acuity and organism type, the association between any vitrectomy and poor final acuity was attenuated, in part because of the type of organism present (Table 6).

\section{Antibiotic Therapies}

The choice of antibiotics used for treatment was known for $82 \%$ of cases $(n=504)$. Among this group, more than $99 \%$ of cases were treated with intravitreal injection of vancomycin, and most also were treated with either ceftazidime $(n=348 ; 69 \%)$ or amikacin $(n=98$; 19\%). Ninety-seven percent of individuals received at least 2 intravitreal antibiotics. Among 
the group treated with vancomycin and amikacin, 26\% had a final acuity of 20/200 or worse, compared with $35 \%$ of the group treated with vancomycin and ceftazidime. Univariate analysis showed a borderline protective effective of amikacin treatment (odds ratio, 0.59; 95\% confidence interval, 0.35-1.00). However, in multivariate analyses, this association was no longer evident (Table 6). Twenty-three percent of cases also were treated with systemic antibiotics. Individuals treated with systemic antibiotics were more likely to have visual acuity of $20 / 200$ or worse at presentation $(P=0.04)$. We did not see an association between the organism cultured and systemic antibiotic use. Individuals treated with systemic antibiotics did not have better outcomes than those not treated with systemic antibiotics, even after controlling for baseline acuity and organism (data not shown).

\section{Final Visual Acuity}

Overall, $43 \%$ of individuals achieved visual acuity of $20 / 40$ or better, whereas $34 \%$ had a final acuity of 20/200 or worse, 26 of whom had visual acuity of NLP. Among the 448 with at least 30 days' of follow-up and a final visual acuity measurement, $48 \%$ achieved $20 / 40$ or better and $29 \%$ of those with at least 30 days follow-up had final acuity of 20/200 or worse. Multiple factors predicted final acuity of 20/200 or worse, with streptococcus infection being the most significant predictor (Table 6). Individuals infected with streptococcus were 11 times more likely to have a final acuity 20/200 or worse, even after adjusting for vitrectomy and acuity at presentation. Gram-negative cases also were more likely to have worse visual outcomes (odds ratio, 4.49; 95\% confidence interval, 1.46-13.79). Worse acuity at presentation was associated with poorer final acuity, whereas vitrectomy did not significantly impact final acuity in this multivariate analysis. Restricting this analysis to those with at least 30 days of follow-up yielded similar results, with slightly stronger associations (Table 6).

\section{Discussion}

To our knowledge, this article reports the largest populationbased series of endophthalmitis cases occurring after cataract surgery to date. Among 615 confirmed cases, $34 \%$ remained legally blind in the affected eye after treatment. Initial visual acuity, age at diagnosis, and causative organism were important predictors of final visual acuity. Vitrectomy use patterns and use of systemic antibiotics differed substantially from EVS guidelines.

\section{Vitrectomy Rates and Outcomes}

Our study evaluated endophthalmitis cases treated in the era after the 1995 release of the EVS results, which provided guidelines for the use of vitrectomy in treating postsurgical endophthalmitis cases. The microbiologic features of the cases we collected from our population-based sample were remarkably similar to those reported by the EVS. More than 90\% of culture-positive cases were gram positive, with the greatest proportion being staphylococci. In the EVS, the use of vitrectomy resulted in a 3-fold improvement in the percentage of patients who achieved 20/40 or better final acuity among those with an initial acuity of LP, with $33 \%$ of patients who underwent vitrectomy having a final acuity of 20/ 40 or better. However, vitrectomy did not improve the chances of good final acuity in cases with presenting acuity better than LP; $66 \%$ of patients undergoing vitrectomy and $62 \%$ 
undergoing a vitreous tap achieved a final acuity of $20 / 40$ or better. ${ }^{4}$ Hence, EVS recommended that vitrectomy be used only for patients with LP and not for those with acuity better than LP. Patients with NLP were excluded from the EVS, so EVS did not make a recommendation for this small group. In our study, $85 \%$ of cases with LP and $41 \%$ of those with initial acuity better than LP were managed with vitrectomy. The rate of vitrectomy in our LP group is similar to that in the Endophthalmitis Population Study of Western Australia (EPSWA) for the population treated during the 5 years after the EVS results $(81 \%)$, whereas the rate of vitrectomy in the group with visual acuity better than LP was much lower in our study than in the EPSWA (41\% vs. $73 \%)^{5}$ and similar to the rates reported in France. ${ }^{7}$

The distribution of initial visual acuity differed somewhat between our study and the EVS, with the EVS group tending to have more severe vision loss. This is likely because the EVS was conducted principally in larger referral centers and was not population based. In our study, only $17 \%$ of cases had LP or worse at presentation compared with $26 \%$ of the EVS population with LP at presentation. Additionally, the proportion with hand movements visual acuity was higher in the EVS (44\% in EVS vs. $34 \%$ in Medicare), whereas our study had a higher proportion of individuals with visual acuity of 20/400 or better (14\% in EVS vs. $28 \%$ in Medicare). ${ }^{4}$ The EPSWA does not provide detailed presenting visual acuity; however, $24 \%$ had vision 20/200 (6/60) or better at presentation, ${ }^{5}$ which is similar to that in our study population.

Interestingly, although both EVS and EPSWA showed the benefit of vitrectomy in the LP group, in our study, vitrectomy did not improve chances of achieving 20/40 final visual acuity in patients with an initial visual acuity of LP, and among those with presenting visual acuity better than LP, individuals undergoing vitrectomy actually fared worse than those who did not undergo vitrectomy. Perhaps this finding simply reflects the association of very poor initial visual acuity and the presence of the most pathogenic organisms, such as streptococci and gram-negative organisms. In such cases, in this population-based sample, no additional benefit of vitrectomy is seen because the retinal destruction had already occurred by the time of vitrectomy. Alternatively, the EVS study excluded patients with NLP visual acuity at presentation, whereas we accepted all cases in our series and analysis. Among the group with initial acuity better than LP, 52\% of those without vitrectomy achieved $20 / 40$ or better acuity compared with $42 \%$ with vitrectomy $(P=0.05)$. A similar proportion of those undergoing immediate vitrectomy and those not undergoing vitrectomy had final visual acuity of 20/200 or worse (22\%), whereas those who underwent delayed vitrectomy ( 2 or more days after diagnosis) had a significantly increased risk of final visual acuity of $20 / 200$ or worse $(51 \% ; P<0.001)$. This difference likely is the result of adverse selection. That is, those undergoing delayed vitrectomy while under observation likely had worsening clinical signs that prompted the treating physician to initiate more aggressive intervention. Importantly, our study is retrospective, and as such, participants were not randomized. Hence, our study was not designed to determine the best treatment approach for an individual, but instead to report on practice patterns.

Across states, the use of vitrectomy varied significantly. Among cases with initial visual acuity better than LP, rates ranged from 19\% in Michigan to 56\% in California, with cases 
in California 9 times more likely to undergo vitrectomy, after adjusting for presenting visual acuity and organism type. Such geographic variability in surgical habit has been observed across many surgical procedures, including cataract surgery. ${ }^{6,8-10}$ One may expect to see less variation at the state level than at smaller units, such as 2 cities, because states have a greater number of opinion leaders and clinical practice offices, such that the wide variation in practice patterns would be averaged across the state. However, our findings suggest nearly a 3-fold variation in vitrectomy use across states. Using the same dataset, we previously examined the rate of cataract surgery by state and found a 2-fold variation. ${ }^{6}$ Among the 5 states included in the current analysis, the rate of cataract surgery ranged from 55 per 1000 person-years in Illinois to 74 per 1000 person-years in Florida. The states with the highest cataract surgery rates (Florida and Michigan) were not the same states that used vitrectomy the most (California and Illinois). This differential finding suggests that the variation in vitrectomy rates across states is not related strictly to lower thresholds for performing procedures in these states. We can only speculate that the reason for this variation may relate to differences at the subspecialty level; however, further research is required to identify the true reasons for this variation.

In the setting of treatment for endophthalmitis, there is no evidence that higher rates of vitrectomy for endophthalmitis are associated with better visual outcomes for those with initial visual acuity better than LP. However, vitrectomy may have some other advantages in cases with initial visual acuity better than LP. As previously described by Krause et al, ${ }^{11}$ advantages of vitrectomy include improved pathogen identification, removal of infecting organisms, and reduction of cells and inflammatory mediators. These advantages need to be weighed against the risk of postoperative complications such as retinal tears, the reduced half-life of intravitreal antibiotic injection that occurs in eyes with vitrectomy, and the morbidity associated with undergoing an additional procedure.

\section{Antibiotic Treatments}

The vast majority of cases were treated with intravitreal injection of vancomycin, and many also were treated with amikacin (22\%) or ceftazidime (71\%). As described above, analyses suggested a borderline positive association between amikacin use and improved probability of vision better than 20/200. However, it is important to note that the design of our study is not optimal to estimate treatment effects of different antibiotics, because there were no standardized treatment algorithms and there are a variety of opportunities for selection bias to affect the findings.

Use of systemic antibiotics was not associated with better visual acuity outcomes. Results of previous studies investigating the role of systemic antibiotics in treating endophthalmitis have been mixed. The EVS reported that systemic antibiotics did not improve visual acuity. ${ }^{4}$ Similarly, a single-center retrospective evaluation of 88 cases in Turkey showed no benefit of systemic antibiotics, ${ }^{12}$ whereas the EPSWA found a nearly 4-fold increased risk of visual acuity worse than $6 / 18$ (equivalent to 20/60) in patients not treated with systemic antibiotics. ${ }^{5}$ However, within that study, $22 \%$ of the 107 eyes included in the multivariate analysis did not receive intravitreal antibiotics for endophthalmitis management, which may alter the benefits of systemic antibiotics significantly. Furthermore, the study includes cases

Ophthalmology. Author manuscript; available in PMC 2016 August 01. 
from 1980 through 2000, so many of the cases were treated before current clinical management practices. ${ }^{5}$ As described in the Turkey-based study, the lack of association between systemic antibiotic use and good visual outcomes in our study does not necessarily mean that systemic antibiotics do not have any role in the management of postoperative endophthalmitis. ${ }^{12}$ Our study was purely observational, and therefore, we cannot make direct inferences regarding the comparative effectiveness of different treatment approaches.

\section{Current Population Compared with That of Other Studies}

Our study population was derived from a representative sample of cataract surgery cases within the United States; hence, it should be representative of postcataract surgery endophthalmitis cases for the United States for the period evaluated. The cataract surgery techniques practiced during the years analyzed remain state of the art today, and treatment for endophthalmitis has not changed in a clinically meaningful way since that time. To our knowledge, the EVS 4 remains the only randomized clinical trial performed in the United States and is still the gold standard for how to treat endophthalmitis. Thus, our study results should be applicable to today's clinical practice. The presenting characteristics of our population are similar to populations in other large-scale multicenter studies, including the EVS $^{4}$ and the Endophthalmitis Population Study of Western Australia 5 (EPSWA) and relatively large single-center studies. ${ }^{13-15}$ Fifty-eight percent of cultured cases showed positive results for at least 1 organism, and coagulasenegative staphylococci was the most commonly recovered organism, representing nearly $50 \%$ of all positive culture results. Streptococci accounted for $12 \%$ of all positive culture results. These results are quite similar to Streptococci accounted for $12 \%$ of all positive culture results. These results are quite similar to staphylococci was recovered from $47 \%$ of cases. ${ }^{5}$ However, the rate of grampositive organisms was slightly higher in our study compared with the EPSWA (93\% vs. $84 \%)$. Other reports from this period show consistent findings among typically Europeanderived populations, ${ }^{4,16}$ whereas 2 studies from Asia report substantially higher rates of gram-positive organisms and a much larger percentage of Pseudomonas infection. ${ }^{17,18}$

\section{Predictors of Poor Outcomes}

In our study, $34 \%$ of patients had a final visual acuity of 20/ 200 or worse. Other studies of this type have reported a similar level of poor outcomes. ${ }^{5,7,19,20}$ Consistent with previous reports, cases involving streptococci were much less likely to have good visual outcomes than cases involving other organisms, $4,5,7,13,16,21,22$ with $70 \%$ of streptococci cases having final visual acuity of counting fingers or worse. This finding highlights the need for optimizing measures to prevent these severe cases, or at least to identify them as early as possible.

Worse initial acuity and older age at diagnosis also were important predictors of poor final acuity. Poor presenting acuity has been reported regularly as a risk factor for poor prognosis. In our study, the risk of poor final acuity increased $15 \%$ for every 5 -year increase in age. Other, smaller studies have not shown a consistent association with age. ${ }^{5,7}$ Whether the lack of association in the other studies is a result of differences in the study populations or because of smaller sample sizes cannot be evaluated with the existing data. We previously reported an increased risk of postcataract surgery endophthalmitis in patients of older 
age. ${ }^{1,23}$ The combined increased risk of endophthalmitis and increased risk of a poor outcome among those in whom endophthalmitis develops makes this age group an important one to focus on for counseling before cataract surgery, closer follow-up, and perhaps use of prophylactic intracameral antibiotics if that technique is not already used routinely. In conclusion, our large, population-based sample of endophthalmitis cases occurring after cataract surgery demonstrated that $93 \%$ were associated with gram-positive organisms. Fortunately, most of these were staphylococci, with relatively favorable outcomes to treatment. Practice patterns related to vitrectomy at the time of diagnosis do not reflect the recommendations of the EVS, which found vitrectomy to be beneficial only for those patients with exceedingly poor vision at presentation. We found that vitrectomy frequently was performed in patients with much better vision at presentation and that the likelihood of a patient undergoing vitrectomy was correlated more with the state of residence than baseline vision. This finding highlights the limited incorporation of clinical trial findings into actual clinical practice. Of course, the best way to confront endophthalmitis is to prevent it in the first place. Future reports will detail the results of our case-control study focusing on identifying risks for endophthalmitis after cataract surgery.

\section{Acknowledgments}

The authors thank the retinal specialists who graciously provided information and the Endophthalmitis Post-cataract Surgery staff for their tireless efforts in data collection.

Supported in part by the National Eye Institute, National Institutes of Health, Bethesda, Maryland (grant no.: R01 EY016769). Dr. Gower is the recipient of an Ernest and Elizabeth Althouse Special Scholars Award from Research to Prevent Blindness, Inc., New York, New York. Dr. Keay was funded by an Australian National Health and Medical Research Council postdoctoral fellowship. (Canberra, Australia).

\section{References}

1. Keay L, Gower EW, Cassard SD, et al. Postcataract surgery endophthalmitis in the United States: analysis of the complete 2003 to 2004 Medicare database of cataract surgeries. Ophthalmology. 2012; 119:914-22. [PubMed: 22297029]

2. Stein JD, Grossman DS, Mundy KM, et al. Severe adverse events after cataract surgery among medicare beneficiaries. Ophthalmology. 2011; 118:1716-23. [PubMed: 21640382]

3. Du DT, Wagoner A, Barone SB, et al. Incidence of endophthalmitis after corneal transplant or cataract surgery in a medicare population. Ophthalmology. 2014; 121:290-8. [PubMed: 23993357]

4. Results of the Endophthalmitis Vitrectomy Study. A randomized trial of immediate vitrectomy and of intravenous antibiotics for the treatment of postoperative bacterial endophthalmitis. Endophthalmitis Vitrectomy Study Group. Arch Ophthalmol. 1995; 113:1479-96. [PubMed: 7487614]

5. Ng JQ, Morlet N, Pearman JW, Constable IJ, et al. Management and outcomes of postoperative endophthalmitis since the endophthalmitis vitrectomy study: the Endophthalmitis Population Study of Western Australia (EPSWA)'s fifth report. Ophthalmology. 2005; 112:1199-206. [PubMed: 15921759]

6. Schein OD, Cassard SD, Tielsch JM, Gower EW. Cataract surgery among Medicare beneficiaries. Ophthalmic Epidemiol. 2012; 19:257-64. [PubMed: 22978526]

7. Combey de LA, Campolmi N, Cornut PL, et al. Baseline factors predictive of visual prognosis in acute postoperative bacterial endophthalmitis in patients undergoing cataract surgery. JAMA Ophthalmol. 2013; 131:1159-66. [PubMed: 23887858]

8. Matlock DD, Groeneveld PW, Sidney S, et al. Geographic variation in cardiovascular procedure use among Medicare feefor-service vs Medicare Advantage beneficiaries. JAMA. 2013; 310:155-62. [PubMed: 23839749] 
9. Jagsi R, Jiang J, Momoh AO, et al. Trends and variation in use of breast reconstruction in patients with breast cancer undergoing mastectomy in the United States. J Clin Oncol. 2014; 32:919-26. [PubMed: 24550418]

10. Jampel HD, Cassard SD, Friedman DS, et al. Trends over time and regional variations in the rate of laser trabeculoplasty in the Medicare population. JAMA Ophthalmol. 2014; 132:685-90. [PubMed: 24744059]

11. Krause L, Bechrakis NE, Heimann H, et al. Incidence and outcome of endophthalmitis over a 13year period. Can J Ophthalmol. 2009; 44:88-94. [PubMed: 19169320]

12. Altan T, Acar N, Kapran Z, et al. Acute-onset endophthalmitis after cataract surgery: success of initial therapy, visual outcomes, and related factors. Retina. 2009; 29:606-12. [PubMed: 19174718]

13. Lalwani GA, Flynn HW Jr, Scott IU, et al. Acute-onset endophthalmitis after clear corneal cataract surgery (1996-2005). Clinical features, causative organisms, and visual acuity outcomes. Ophthalmology. 2008; 115:473-6. [PubMed: 18067969]

14. Cornut PL, Thuret G, Creuzot-Garcher C, et al. Relationship between baseline clinical data and microbiologic spectrum in 100 patients with acute postcataract endophthalmitis. Retina. 2012; 32:549-57. [PubMed: 21968507]

15. Mollan SP, Gao A, Lockwood A, et al. Postcataract endophthalmitis: incidence and microbial isolates in a United Kingdom region from 1996 through 2004. J Cataract Refract Surg. 2007; 33:265-8. [PubMed: 17276268]

16. Simunovic MP, Rush RB, Hunyor AP, Chang AA. Endophthalmitis following intravitreal injection versus endophthalmitis following cataract surgery: clinical features, causative organisms and posttreatment outcomes. Br J Ophthalmol. 2012; 96:862-6. [PubMed: 22446145]

17. Ding Y, Lin M, Liu H, et al. Outcomes of post-cataract surgery endophthalmitis referred to a tertiary center from local hospitals in the south of China. Infection. 2011; 39:451-60. [PubMed: 21710121]

18. Cheng JH, Chang YH, Chen CL, et al. Acute endophthalmitis after cataract surgery at a referral centre in Northern Taiwan: review of the causative organisms, antibiotic susceptibility, and clinical features. Eye (Lond). 2010; 24:1359-65. [PubMed: 20379208]

19. Gupta A, Orlans HO, Hornby SJ, Bowler IC. Microbiology and visual outcomes of culture-positive bacterial endophthalmitis in Oxford, UK. Graefes Arch Clin Exp Ophthalmol. 2014; 252:1825-30. [PubMed: 25028312]

20. Karacal H, Kymes SM, Apte RS. Retrospective analysis of etiopathogenesis of all cases of endophthalmitis at a large tertiary referral center. Int Ophthalmol. 2007; 27:251-9. [PubMed: 17437060]

21. Barry P, Gardner S, Seal D, et al. Clinical observations associated with proven and unproven cases in the ESCRS study of prophylaxis of postoperative endophthalmitis after cataract surgery. $\mathrm{J}$ Cataract Refract Surg. 2009; 35:1523-31. [PubMed: 19683148]

22. Al-Mezaine HS, Kangave D, Al-Assiri A, Al-Rajhi AA. Acute-onset nosocomial endophthalmitis after cataract surgery: incidence, clinical features, causative organisms, and visual outcomes. J Cataract Refract Surg. 2009; 35:643-9. [PubMed: 19304084]

23. West ES, Behrens A, McDonnell PJ, et al. The incidence of endophthalmitis after cataract surgery among the U.S. Medicare population increased between 1994 and 2001. Ophthalmology. 2005; 112:1388-94. [PubMed: 15953637]

\section{Abbreviations and Acronyms}

$\begin{array}{ll}\text { CNS } & \text { coagulase-negative staphylococci } \\ \text { EPSWA } & \text { Endophthalmitis Population Study of Western Australia } \\ \text { EVS } & \text { Endophthalmitis Vitrectomy Study } \\ \text { LP } & \text { light perception }\end{array}$



no light perception 


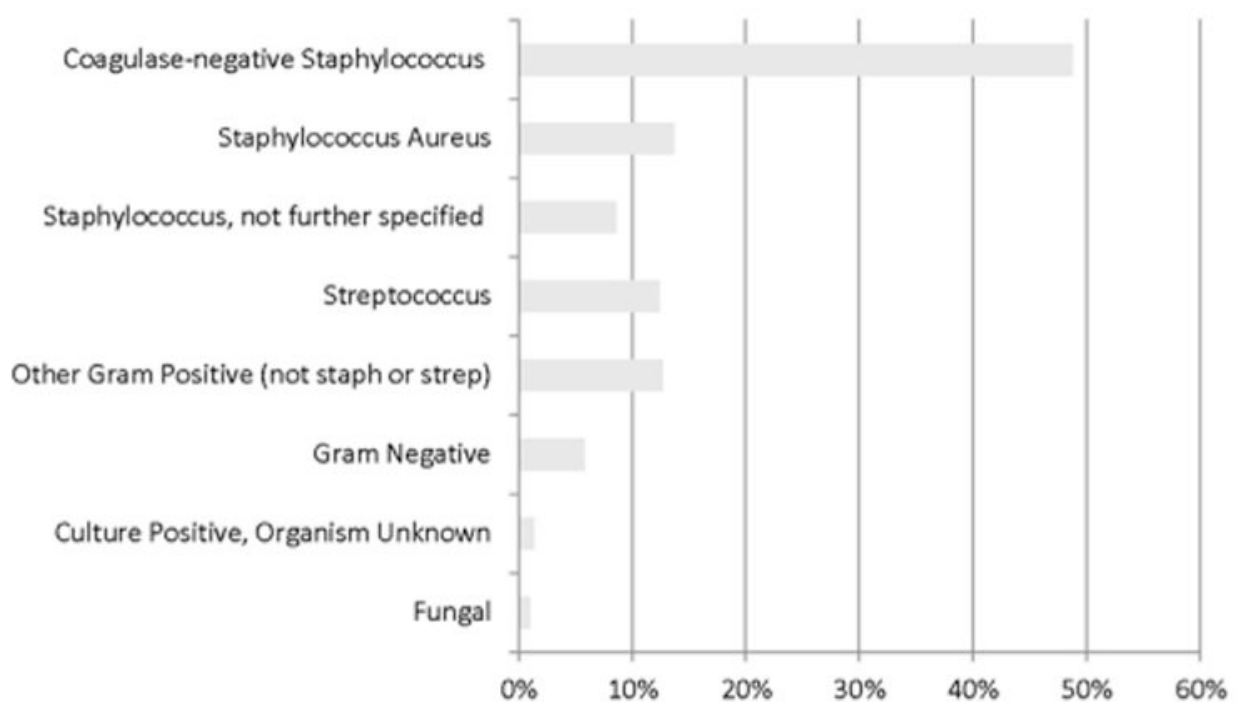

*12 cultures grew multiple organisms.

Figure 1.

Graph showing the percent of cultures growing each organism, from among 291 cultures with positive results. 
Table 1

Characteristics of 615 Patients with Confirmed Endophthalmitis

\begin{tabular}{lr}
\hline Characteristic & No. $(\%)$ \\
\hline Female gender & $351(57.1)$ \\
Age (yrs) & \\
$65-74$ & $202(32.9)$ \\
$75-84$ & $322(52.4)$ \\
$85+$ & $91(14.8)$ \\
State & \\
California & $153(24.9)$ \\
Florida & $498(32.2)$ \\
Illinois & $93(15.1)$ \\
Michigan & $67(10.9)$ \\
Texas & $104(16.9)$ \\
Year & \\
2003 & $352(57.2)$ \\
2004 & $263(42.8)$ \\
Initial acuity & \\
Light perception or no light perception & $100(17.1)$ \\
Hand movements & $26(4.2)$ \\
Counting fingers & \\
$20 / 200-20 / 400$ & $201(34.4)$ \\
$20 / 50-20 / 100$ & $122(20.9)$ \\
$20 / 40$ or better & $80(13.7)$ \\
Time from surgery to endophthalmitis diagnosis (days) & \\
0 & $65(11.1)$ \\
$1-3$ & $17(2.9)$ \\
$4-7$ & \\
$8-14$ & \\
$15-28$ & \\
& \\
& \\
&
\end{tabular}

Ophthalmology. Author manuscript; available in PMC 2016 August 01. 
Table 2

Association between Initial Acuity and Culture Results

\begin{tabular}{|c|c|c|c|c|}
\hline \multirow[b]{2}{*}{ Initial Snellen Acuity } & \multirow[b]{2}{*}{ Overall } & \multicolumn{3}{|c|}{ Culture Status } \\
\hline & & Positive & Negative & Unknown \\
\hline Total & 615 & 291 & 211 & 113 \\
\hline Light perception or no light perception & 100 & $71(71.0)$ & $17(17.0)$ & $12(12.0)$ \\
\hline Hand movements & 201 & $111(55.2)$ & $54(26.9)$ & $36(17.9)$ \\
\hline Counting fingers & 122 & $51(41.8)$ & $50(41.0)$ & $21(17.2)$ \\
\hline $20 / 200-20 / 400$ & 80 & $26(32.5)$ & 39 (48.8) & $15(18.8)$ \\
\hline $20 / 50-20 / 100$ & 65 & $16(24.6)$ & 37 (56.9) & $12(18.5)$ \\
\hline $20 / 40$ or better & 17 & $6(35.3)$ & $7(41.2)$ & $4(23.5)$ \\
\hline Unknown & 30 & $10(33.3)$ & $7(23.3)$ & $13(43.3)$ \\
\hline
\end{tabular}

Data are no. (\%) unless otherwise indicated. 
Table 3

Time of First Vitrectomy Overall and by Presenting Acuity*

\begin{tabular}{lrrrr}
\hline & \multicolumn{4}{c}{ Time between Diagnosis and Vitrectomy } \\
\cline { 2 - 5 } Initial Visual Acuity & No Vitrectomy & Day 0 or Day 1 & Days 2-7 & Day 8+ \\
\hline No light perception & $4(57.1)$ & $3(42.9)$ & 0 & 0 \\
Light perception & $13(14.8)$ & $67(76.1)$ & $5(5.7)$ & $3(3.4)$ \\
Hand movements & $93(49.7)$ & $61(32.6)$ & $8(4.3)$ & $25(13.4)$ \\
Counting fingers & $73(62.9)$ & $29(25.0)$ & $5(4.3)$ & $9(7.8)$ \\
20/200-20/400 & $48(65.8)$ & $20(27.4)$ & $1(1.4)$ & $3(5.5)$ \\
20/50-20/100 & $44(71.0)$ & $13(21.0)$ & $0(0.0)$ & $5(8.1)$ \\
20/40 or better & $11(78.6)$ & $3(21.4)$ & $0(0.0)$ & $0(0.0)$ \\
Overall & $295(51.4)$ & $211(36.8)$ & $19(3.3)$ & $49(8.5)$
\end{tabular}

Data are no. (\%).

Sixty-eight missing vitrectomy status, date of vitrectomy, presenting acuity, or a combination thereof and are excluded from the vision-specific lines. Forty-one are missing vitrectomy status information and are excluded from the overall category. 

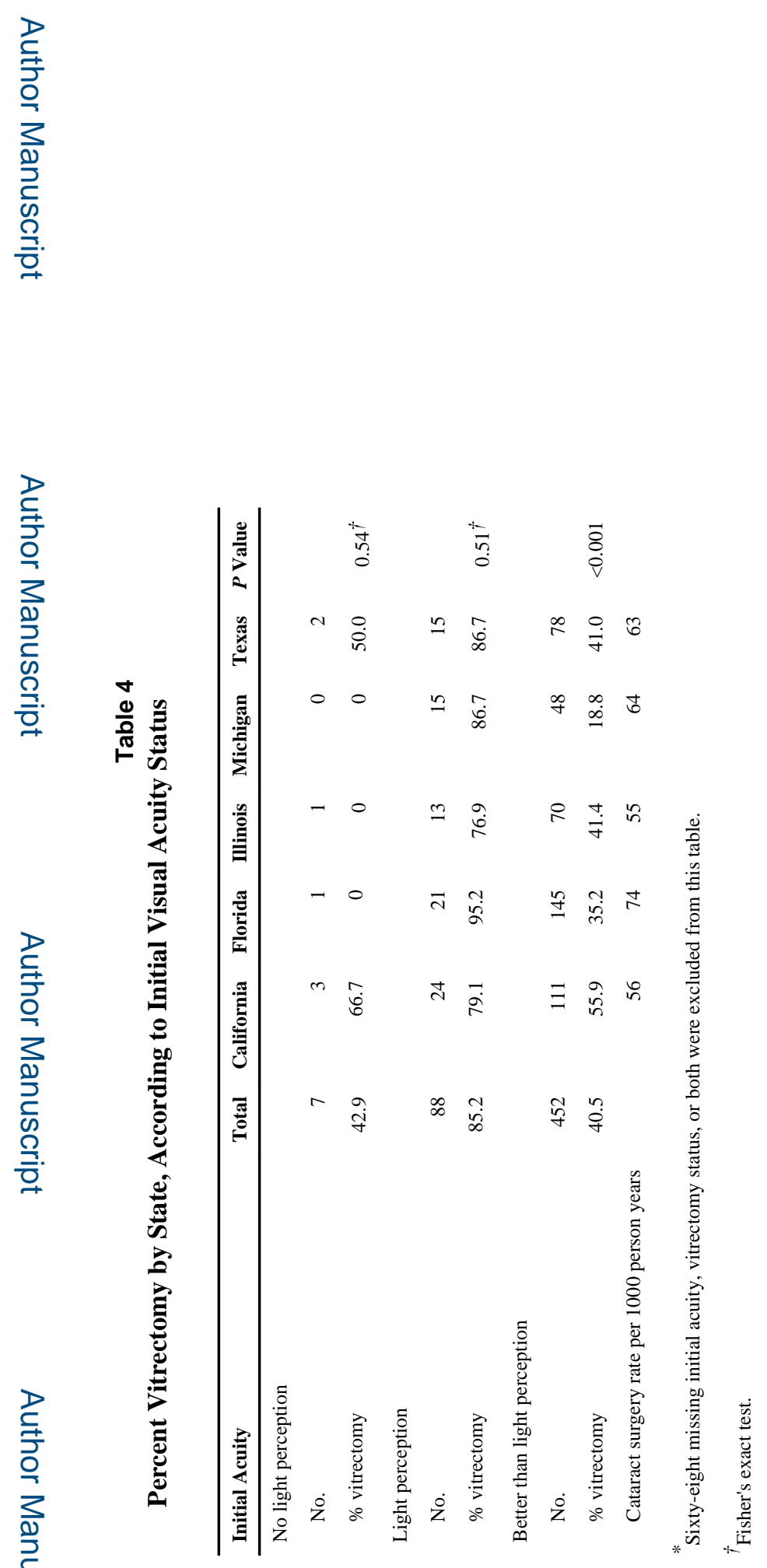

Ophthalmology. Author manuscript; available in PMC 2016 August 01. 

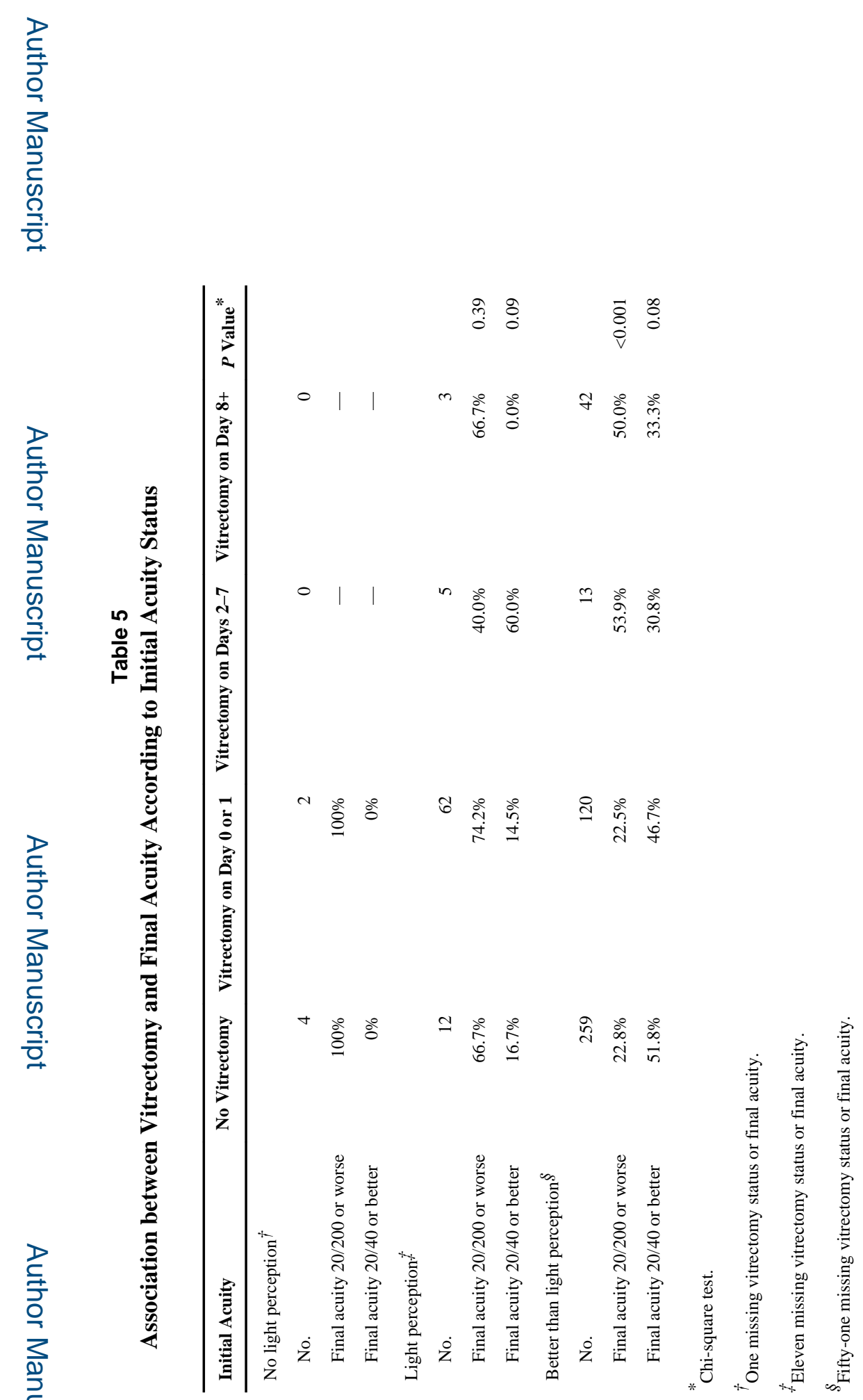

Ophthalmology. Author manuscript; available in PMC 2016 August 01. 


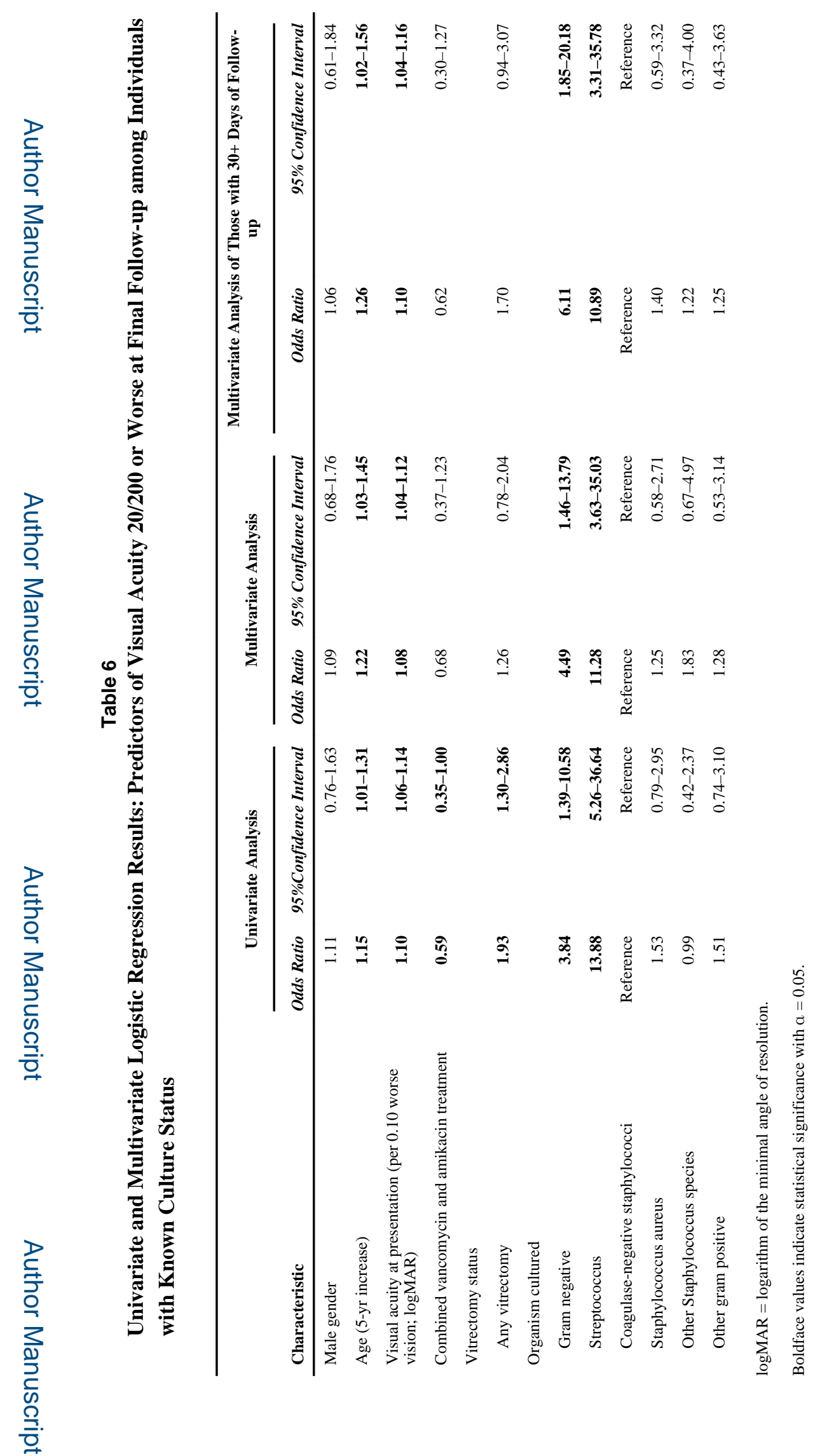

Ophthalmology. Author manuscript; available in PMC 2016 August 01. 